\title{
A review of David Elliot's clinical procedures in primary eye care
}

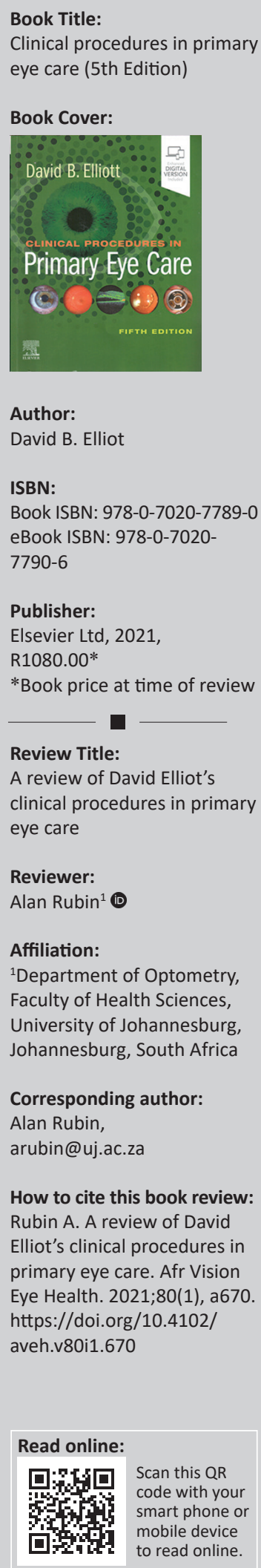

This well-referenced and amply illustrated book (Clinical Procedures in Primary Eye Care), now in its fifth edition after its first publication in 1997, is primarily aimed towards developing the knowledge and clinical skills of undergraduate students in optometry. However, students in ophthalmology will also find the book useful, and many practitioners in both optometry and ophthalmology would also find material of interest and value, especially some chapters that discuss topics such as evidence-based science relating to eye and vision examination and regarding modern ophthalmic procedures such as corneal topography and optical coherence tomography (with a substantial increase in content in the latest edition). Content on several topics such as ocular surface disorders and myopia and their management or treatment have been included or expanded upon in this latest edition. I particularly enjoyed reading Chapter 8 of the book (by Professors Elliot and Pesudovs) that discussed variations of the normal eye together with many high-quality colour photographs. Most eye-related books (or eBooks) emphasise the diseased eye, and perhaps a future book concerning only the normal eye and its many variations might be something to consider for those aspiring to become authors of such clinical and professional resources. Another important addition to this 5th edition is a section concerning mini-scleral and scleral contact lenses that have become important in the treatment of various clinical problems, especially keratoconus where advances in lens design and materials have steadily increased the popularity and potential applications for such lenses.

The editor and author of Clinical Procedures in Primary Eye Care, David Elliot, is a Professor of Clinical Vision Science, at the School of Optometry and Vision Science, University of Bradford, Yorkshire, United Kingdom, and until recently he was also the editor of the highly regarded journal Ophthalmic and Physiological Optics. Professor Elliot has extensive knowledge, experience and a unique perspective embracing both clinical and theoretical aspects in optometry and vision science, and this is clearly evident through a careful reading of the contents of this book. The book and its various chapters are further enhanced by inputs from other contributors from the United Kingdom, the United States, Canada, Japan and Australia. The book also has many accompanying videos (available to the book purchasers) that are well-produced, interesting and generally very useful, particularly for students of optometry (and ophthalmology) in terms of aspects such as communication skills, for example during case history and during the performance and application of essential vision and ocular techniques. For instance, the digital content about the relatively simple process of inserting and removing a scleral lens would be especially helpful for students starting out on their academic and clinical education, and certainly practitioners, whether young or a little older, will also find many of these videos insightful and of assistance in better understanding many of the topics concerned.

Over recent years, there have been significant improvements in technology in relation to eye and vision assessment, and many of these advancements in clinical practice such as ultra-wide field retinal imaging are included in this book; some of which are important also in terms of telemedicine, as such methods will achieve greater application in the years to come.

A chapter (by Professor Patricia Hrynchak of the School of Optometry and Vision Science at the University of Waterloo, Canada), albeit short, involving procedures such as carotid artery auscultation in physical examination of patients with transient monocular blindness (amaurosis fugax), retinal emboli or other retinal lesions such as haemorrhages or dilated tortuous veins (as with venous stasis retinopathy) is nicely illustrated with many useful hints so as to avoid common errors when performing and interpreting results with such procedures. Also for palpation of lymph nodes, there are two videos that are useful, but perhaps in future editions video content relating to this specific section (Chapter 9) could be extended a little, as it is potentially an area

Copyright: @ 2021. The Author(s). Licensee: AOSIS. This work is licensed under the Creative Commons Attribution License. 
where many individuals in different parts of the world may lack experience or exposure to such procedures that are of a more general nature involving clinical medicine. Students, and even sometimes more experienced clinicians, are often uncertain about such procedures, and video and digital instruction could be very helpful in remedying this possible limitation.

In this book, Chapter 2, by Professor Elliot, emphasises communication skills as an essential part of the examination of patients, and this can often be an area where improvements can be extremely useful in easily and quickly enhancing patient and clinician understanding and inter-professional communications, whether through better record keeping or proper preparation of clinical reports, and both students and practitioners often fail to achieve what is truly desirable in such areas. Small improvements in such areas sometimes can produce disproportionately large improvements in terms of patient satisfaction, interpersonal and professional interactions and can even make the difference between more or less successful clinical practices and greater intra-personal professional satisfaction and endeavour.
The information provided in this book is concise and relevant, and readers should find the book very helpful and stimulating; this book is a very useful general resource for students who may be studying one or more of the eye-care disciplines. Practitioners would, no doubt, also benefit from the perusal of the contents of the various chapters, and many useful clinical tips, sensible suggestions and advice are provided with vital concepts clearly explained.

A comprehensive subject index and adequate referencing are provided for the various topics described in the text, and I would not hesitate in recommending this book to optometrists, ophthalmologists, optical dispensers, ophthalmic nurses and students in these fields who need to more thoroughly understand ocular and vision assessment and the general management and treatment of common disorders involving the ocular system. The book with its accompanying videos and eBook is relatively inexpensive and more importantly is enjoyable reading whilst being deeply informative and beneficial for knowledge development and towards improving the process of working with patients in everyday clinical practice. 